\title{
The Adiabatic Electron Plasma and Its Equation of State
}

\section{G. Knorr}

and

J. Nuehrenberg

\section{Department of Physics and Astronomy The University of Iowa Iowa City, Iowa 52240}

May 1970

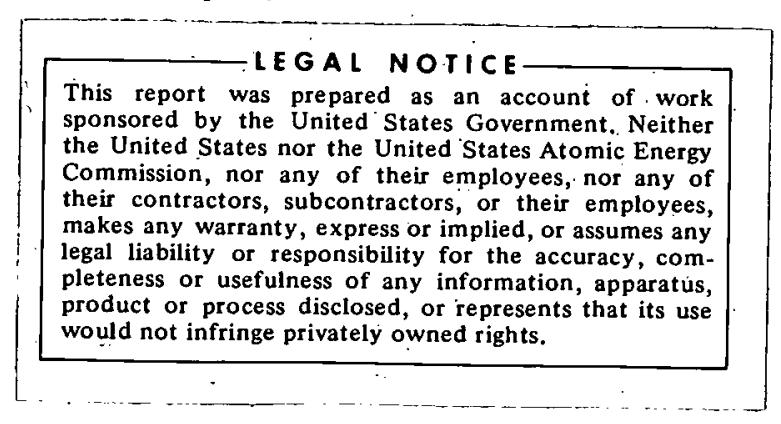

This work was supported in part by Atomic Energy Commission Grant No. AT(11-1)-2059. 


\section{DISCLAIMER}

This report was prepared as an account of work sponsored by an agency of the United States Government. Neither the United States Government nor any agency Thereof, nor any of their employees, makes any warranty, express or implied, or assumes any legal liability or responsibility for the accuracy, completeness, or usefulness of any information, apparatus, product, or process disclosed, or represents that its use would not infringe privately owned rights. Reference herein to any specific commercial product, process, or service by trade name, trademark, manufacturer, or otherwise does not necessarily constitute or imply its endorsement, recommendation, or favoring by the United States Government or any agency thereof. The views and opinions of authors expressed herein do not necessarily state or reflect those of the United States Government or any agency thereof. 


\section{DISCLAIMER}

Portions of this document may be illegible in electronic image products. Images are produced from the best available original document. 


\section{ABSTRACT}

For slow processes in a collisionless two-component plasma which conserve the adiabatic invariant of the electrons, the equation of motion for electrons is derived. The conservation laws for the whole system are discussed in relation to the equation of state and a consistent distribution function for the electrons. 


\section{INTRODUCTION}

When slow oscillations in a one dimensional, two component plasma are investigated, one often describes the electrons collectively by an equation of state rather than by a more detailed equation of motion. Thus for example, BERNSTEIN AND TREHAN (1960) assume thermal equilibrium for the electrons, such that

$$
n_{e}=n_{0}\left[\exp \left(e \varphi / \theta_{e}\right)-1\right],
$$

where $\mathrm{n}_{e}$ and $\mathrm{n}_{o}$ are the disturbed and undisturbed electron density, $\varphi(x, t)$ the potential, $A_{e}$ the electron temperature and -e the electron charge. Eq. (1) is assumed to be satisfied at any instance. MONTGOMERY (1967) assumes that the electron distribution function f remains proportional to

$$
\exp \left[-\left(\frac{1}{2} m v^{2}-e \varphi\right) / \theta_{e}\right]
$$

which is even a more stringent condition than Eq. (I) and reduces to it after integration over velocity space. 
In the numerical simulation of plasmas the question of the validity of an equation of state for electrons becomes particularly important (e.g. MASON 1969). When we follow ion and electron trajectories on a computer, the time step must be adjusted in such a way that a plasma oscillation can still be represented. This means however, that we spend most of our computing effort on the time scale of the electrons. Following the evolution of an ion accoustic wave, for example, becomes either extremely time consuming or practically impossible. It is customary to represent the electrons by an equation of state (Compare ARMSTRONG et al.). Then the time step can be adjusted to the slow ion motion and the electron motion is taken care of by the equation of state. Usually the latter is introduced without much justification and the question arises, how good this approximation really is. HARDING (1968) has investigated this problem numerically for certain special cases. The physical basis of Eq. (1) and (2) seems to be that a Maxwellian distribution is always maintained, e.g. by electron collisions. This means that $\nu_{\text {ee }} \tau \ll I$ where $\nu_{e e}$ is the electron electron collision rate and $\tau$. is the characteristic time scale of the process in question.

When the electron gas is collision free, Eq. (1) and (2) become questionable. However, we know [see e.g. FUES (1927)] that for a spacially periodic geometry the adiabatic invariant of electrons is conserved, if the potential change (like in ion waves) is slow in a certain sense. 
In the following we trace some consequences of the assumption of adiabaticity for the equation of motion and an assumed equation of state for a one dimensional electron plasma. 
II. THE ADIABATIC ELECTRON DISTRIBUTION

We consider processes in a plasma whose time scale is much slower than the time scale of the electron motion. An example is ion waves. We can then describe the electron distribution as being quasistatic. A static electron distribution depends on the total energy $W$

$$
W=\frac{1}{2} m v^{2}-e \varphi(x, t)
$$

only, so we may write $f_{e}=f_{e}(w, t)$. Under these conditions the adiabatic invariant

$$
J=\oint \quad v d x=\oint \frac{\sqrt{\frac{2}{m}[W+e \varphi(x, t)]}}{m} d x
$$

is conserved. The integral has to be taken from turning point to tuming point for trapped particles and over a periodicity interval for free particles. The adiabaticity assumption can be expressed as

$$
\frac{\partial}{\partial t} f_{e}(J, t)=0
$$

(We omit the dichotomic variable $\sigma= \pm 1$ representing velocity direction for free particles and justify this in sec. III.) 
In order to estimate its validity we consider ion waves whose frequency is roughly given by: $\omega_{i}=\left(\theta_{e} / M\right)^{1 / 2} k$ where $M$ is the ion mass and $k$ the wave number of the wave. For trapped particles, the bounce frequency $u_{3}$ is of order $(e \varphi / m)^{l / z_{k}}$ so that

$$
w_{b} / w_{i}=\left[\left(e \varphi / \theta_{e}\right)(M / m)\right]^{I / 2} .
$$

If the potential of the wave is large, say $1 / 10$ of $\mathrm{A}_{e}$ or more, $\omega_{b} / \omega_{i} \gg 1$, which means that the adiabaticity assumption is satisfied for most of the trapped particles. If $e_{\varphi} / A_{e}$ is very small, so that $\omega_{b} / \omega_{i}$ is of order one, the adiabaticity assumption does not hold. However, in this case the number of trapped particles is negligible. For free particles, the bounce frequency is of the order $k\left(\theta_{e} / m\right)^{1 / 2}$ and the ratio becomes

$$
w_{b} / w_{i}=(M / m)^{1 / 2} \gg 1 \text {. }
$$

Thus, for most electrons the adiabaticity assumption is satisfied, except for those which are almost untrapped or almost trapped. The number of these particles however, should generally be small.

We want to describe now the electron distribution in terms of the energy $W$ and time, $f_{e}=f_{e}(W, t)$. Along two trajectories, 1 and $?$, of particles with slightly different energies (see Fig. 1) 
the distribution function is constant. When the potential changes in time, curves $I$ and 2 are mapped into $I^{\prime}$ and $2^{\prime}$, the area between the curves remaining invariant because $\mathrm{J}$ is invariant. Thus, the number of particles between the two curves remains invariant, but their energy has changed. We can therefore write

$$
f(W+\Delta W, t+\Delta t)=f(W, t),
$$

or in differential form

$$
\frac{\partial f(W, t)}{\partial t}=-\frac{\Delta W}{\Delta t} \frac{\partial f(W, t)}{\partial W}
$$

$\Delta W$ represents the energy a particle has gained in the time $\Delta t$ where $\Delta t$ comprises many bounce periods. So, if we introduce

$$
\frac{d \bar{W}}{d t}=\frac{\Delta W}{\Delta t}
$$

for the energy change of a particle along its trajectory, we can integrate Eq. (8):

$$
f_{e}=f_{e}[W-\bar{W}(t)+\bar{W}(0)],
$$


where, since $J$ is a constant along a trajectory, $\bar{W}(t)$ is given by

$$
\oint \sqrt{W(0)+\operatorname{req}(x, 0)} d x=\oint \sqrt{W(t)+\operatorname{erp}(x, t)} d x .
$$

The meaning of Eq. (9) is the following: In order to calculate the value of $f_{e}$ at time $t$ for a certain $W$, we choose $\bar{W}(t)=W$ and evaluate the corresponding $\bar{W}(0)$ from Eq. (10). $f(\bar{W}(0))$ is then the desired value. Eqs. (9) and (10) constitute the equation of motion for an adiabatic electron gas. This solves the problem of the integration of the motion of the electrons on the slow time scale. The electron density, which is required for Poisson's equation to make the electron-ion system self consistent, is obtained by another integration. A computer solution of Eqs. (9) and (10) is still quite involved. It is time consuming because for every time step, Eq. (10) and the density have to be evaluated. In the appendix, an analytical solution of Eqs. (9) and (10) is given for a special case.

As is evident from Eq. (9) the density will in general always depend explicitly on the time:

$$
n_{e}(x, t)=n_{e}[\varphi(x, t), t]
$$


More precisely, because $\bar{W}(t)$ is a functional of $\varphi(x, t)$, we can write alternatively

$$
n_{e}(x, t)=n_{e}[\varphi(x, t), \lambda(\varphi)],
$$

where the functional $\lambda$ of $\varphi$ depends on time.

We continue and discuss the conservation laws for the whole ion-electron system in relation to the equation of state and a consistent distribution function for the electrons. 
III. EQUATION OF STATE

When most electrons are untrapped, one might try to expand Eq. (10) in powers of $e \varphi / W$ and use the result in Eq. (9). To lowest significant order we obtain

$$
\bar{W}(t)-\bar{W}(0)=\frac{1}{4} e^{2} / L\left[\frac{\int_{0}^{L} \varphi^{2}(x, 0) d x}{\bar{W}(0)}-\frac{\int_{0}^{L} \varphi^{2}(x, t) d x}{\bar{W}(t)}\right] .
$$

Thus Eq. (9) yields to first order in $e \varphi / w$

$$
f(W, t)=f(W, o)
$$

For a Maxwellian, we obtain the result quoted in Eq. (2), which, therefore, represents an adiabatic distribution function up to first order in $e \varphi / w$. We then would have

$$
n_{e}(x, t)=n_{e}[\varphi(x, t)] .
$$

Such an equation of state does in general not even conserve the total number of electrons correctly. In order to conserve particles for periodic boundary conditions, Eq. (14) may be used only in its linearized form: 


$$
n_{e}(x, t)=n_{0}(1+\beta e \varphi(x, t)) .
$$

We return to the more general equation of state (11) and ask if the integral conservation theorems of the ion-electron system impose any restrictions on the form of the equation of state. We show that except for particle conservation this is not the case.

The number of electrons is evidently always conserved if we write

$$
n_{e}(x, t)=g(\varphi(x, t), t) /\langle g(\varphi(x, t), t)\rangle,
$$

where \langle\rangle denotes the spatial average (we always assume periodic boundary conditions), and $g(\varphi, t)$ is an arbitrary function of $\varphi$ and $t$.

Next we consider momentum and energy conservation. Using the ion Vlasov equation, Poisson's equation, and an arbitrary electron density $\mathrm{n}_{\mathrm{e}}(\mathrm{x}, \mathrm{t})$, one can easily show that momentum and energy conservation can be written in the form

$$
\begin{aligned}
& \frac{d}{d t}\left(J_{i}+J_{e}\right)=0 \\
& \frac{d}{d t}\left(w_{i}+w_{e}+\frac{1}{8 \pi}\left\langle E^{2}\right\rangle\right)=0 .
\end{aligned}
$$


The quantities $(d / d t) J_{i}$ and $(d / d t) W_{i}$ are calculated from the ion Vlasov equation and are

$$
\begin{aligned}
& \frac{d}{d t} J_{i}=\frac{d}{d t} m_{i}\left\langle\int_{-\infty}^{\infty} v f_{i} d v\right\rangle=e\left\langle E n_{i}\right\rangle \\
& \frac{d}{d t} W_{i}=\frac{d}{d t} \frac{m_{i}}{2}\left\langle\int_{-\infty}^{\infty} v^{2} f_{i} d v\right\rangle=-e\left\langle\varphi \frac{\partial n_{i}}{\partial t}\right\rangle .
\end{aligned}
$$

Consequently, the time derivatives of the electron momentum and energy must be defined as

$$
\begin{aligned}
& \frac{d}{d t} J_{e}=-e\left\langle E n_{e}\right\rangle \\
& \frac{d}{d t} W_{e}=e\left\langle\varphi \frac{\partial n_{e}}{\partial t}\right\rangle .
\end{aligned}
$$

Since this holds for any electron density $n_{e}(x, t)$, momentum and energy conservation are then satisfied for any equation of state of type (16) if we use Eqs. (19) as definitions for momentum and energy.

In order to obtain an equation of state which is not completely arbitrary, we therefore have to resort to a microscopic model. The electron distribution function has to be specified such that Eqs. (19 a,b) are satisfied, where momentum, energy, and equation of state are calculated from the distribution function. 
For example, one can easily show explicitly that no distribution function of the type

$$
f_{e}(W)=h(\varphi) \widetilde{f}\left[\frac{1}{2} m_{e} v^{2}-e \varphi(x, t)\right]
$$

satisfies Eq. (19 b) although, with

$$
h(\varphi)=\left[\left\langle\int_{-\infty}^{\infty} \widetilde{f} d v\right\rangle\right]^{-1},
$$

particles are conserved. We obtain

$$
\frac{d}{d t} w_{e}=\frac{1}{2}\left\langle n_{e} \frac{\partial e \varphi}{\partial t}\right\rangle+\left\langle\int_{-\infty}^{\infty} \frac{l}{2} m_{e} v^{2} \tilde{f} d v\right\rangle \cdot \frac{d}{d t} h(\varphi)
$$

which is in general different from Eq. (19 b). A trivial example is a Maxwell-Boltzman distribution with constant temperature for which the kinetic energy is constant in time.

The simplest example of a distribution function which satisfies Eq. (19 b) is a Maxwell-Boltzman distribution with time dependent temperature:

$$
f_{e}=n_{0}\left(2 \pi A / m_{e}\right)^{-\frac{1}{2}} \exp \left[-\left(\frac{1}{2} m_{e} v^{2}-\operatorname{e} \varphi(x, t)\right) / \theta\right] /\langle\exp (e \varphi / A)\rangle .
$$

The equation of state

$$
\mathrm{n}_{\mathrm{e}}=\mathrm{n}_{\mathrm{o}} \exp (e \varphi / \theta) /\langle\exp (e \varphi / \theta)\rangle
$$


is the form of Eq. (16); the total energy is given by $\frac{1}{2} \theta n_{0}$ and obeys according to Eq. (19 b)

$$
\frac{I}{2} n_{0} \frac{d}{d t} A=e\left\langle\varphi \frac{\partial n_{e}}{\partial t}\right\rangle
$$

This shows that the temperature is a functional of the potential and the density is of the form of Eq. (12). On account of this example, it is evident that Eg. (12) is more than a minor modification of Eq. (14), at least in those cases in which the energy exchange of the electrons with the ions and the electric field plays a significant role. If we consider the linear case $\mathrm{e} \varphi / \theta \ll 1$, we of course recover Eq. (15). $\beta=1 / A$ is a constant as it should be, since the time derivative of $\theta$ is of second order in $e \varphi / \theta$, which is seen from Eq. (20).

Another distribution function consistent with Eq. (19 a) is the adiabatic electron distribution of Sec. II, because the energy change of each electron is treated correctly in the limit that its adiabatic invariant is a constant.

Let us now consider the momentum equation (19 a). For a spatially periodic system without charge accumulation at $\pm \infty$, we have $\langle E\rangle=0$. Because of Maxwell's equation $(d / d t) E=-\frac{4 \pi}{c}\left(j_{i}+j_{e}\right)$, ion and electron momenta are then related by

$$
\frac{1}{m_{i}} J_{i}=\frac{1}{m_{e}} J_{e} \text {. }
$$


Together with Eq. (17), we see that both momenta are constant in time. This is in accordance with the definition of the electron momentum Eq. (19 b), since $\left\langle E n_{e}\right\rangle=0$ for any equation of state if $\langle E\rangle=0$. Thus, we may choose $J_{e}=0$; however, the dichotomic variable $\sigma= \pm 1$ mentioned in Sec. II can, in general, give rise to a time dependent electron momentum. Therefore, this variable can be omitted for a choice of a distribution function which is consistent with an equation of state and momentum conservation. 


\section{APPENDIX}

As an example, we consider an adiabatic distribution for a single wave with wave number $k$ and time dependent amplitude $\varphi_{0}(\mathrm{t}):$

$$
\varphi(x, t)=\varphi_{0}(t) \cos k x
$$

Numerical experiments indicate that this is a good approximation for certain cases. The invariant Eq. (4) can be calculated explicitly and leads to complete elliptic integrals of first kind, $\mathrm{K}(x)$, and of second kind, $\mathrm{E}(x)$. The result can be written for free particles as

$$
J=(2 / k)\left[W+\varphi_{0}(t)\right]^{1 / 2} E(x),
$$

and for trapped particles as

$$
J=(2 / k)\left[W+\varphi_{0}(t)\right]^{I / 2} x\left[E(I / x)+\left(I / x^{2}-I\right) K(I / x)\right],
$$

where 


$$
x^{2}=2 \varphi_{0}(t)\left[w+\varphi_{0}(t)\right]^{-1}=2 \varphi_{0}(t)\left[\frac{1}{2} v^{2}+\varphi_{0}(t)(1-\cos k x)\right]^{-1} .
$$

According to Eq. (5) the distribution function can be written as

$$
f=f\left[2 \varphi_{0}(t) E^{2}(x) / x^{2}\right], x^{2}<1 \text { for free particles }
$$

and

$$
f=f\left\{2 \varphi_{0}(t)\left[E(1 / x)+\left(1 / x^{2}-1\right) K(1 / x)\right]^{2}\right\}, x^{2}>1 \text { for }
$$

trapped particles.

Note that for $x^{2} \rightarrow 1$ the arguments become the same for both cases and $f$ is a continuous function of $x$ and $v$.

In the limit $x^{2} \rightarrow 0$ we obtain $f=f(W)$ as in Eq. (12) and in the limit $x^{2}-\infty$ we find $f=f\left(\frac{W+\varphi_{0}(t)}{\sqrt{\varphi_{0}(t)}}\right) \cdot W+\varphi_{0}(t)$ is the appropriately normalized energy; $\sqrt{\varphi_{O}(t)}$ is proportional to the bounce frequency of a particle. Thus, the argument of $f$ is proportional to the invariant of an harmonic oscillator, as it should be.

Integrating the distribution function over velocity space; we obtain for the density 


$$
n_{e}=n_{e}\left[\varphi_{0}(t), \varphi(x, t) / \varphi_{0}(t)\right],
$$

which we can write as $n_{e}=n_{e}[\varphi(x, t), t]$ in accordance with Eq. (11). In this particular case we can also write it as $n_{e}=n_{e}\left[\varphi_{0}(t), \cos k x\right]$. 
Armstrong T.P., Harding R.C., Knorr G. and Montgomery D. (1970) in: Methods in Computational Physics, Volume 9, B. Alder, S. Fermbach and M. Rotenberg, Eds. Academic Press, New York, 1970. Chapter IV.

Bernstein I. B. and. Trehan S.K. (1960) Nucl. Fusion, I, 3.

Fues E. (1927) Handbuch der Physik, Springer, Berlin, 1927. Volume V, Chapter 4.

Harding, R.C. (1968) Phys. Fluids 11, 2233.

Mason R.J. (1969) in: Third Annual Numerical Plasma Simulation Conference, Sept. $2-5$, 1969, Stanford University. Montgomery D. C. (1967), Phys. Rev. Letters 19, 1465. 
Figure 1. Trajectories of trapped and untrapped electrons in phase space. Trajectories $I$ and 2 are mapped into curves $I^{\prime}$ and $2^{\prime}$ at a later time due to variation of the electrostatic potential. The area between curves 1 and 2 is equal to the area between $I^{\prime}$ and $2^{\prime}$. 


\section{A- G70-27}

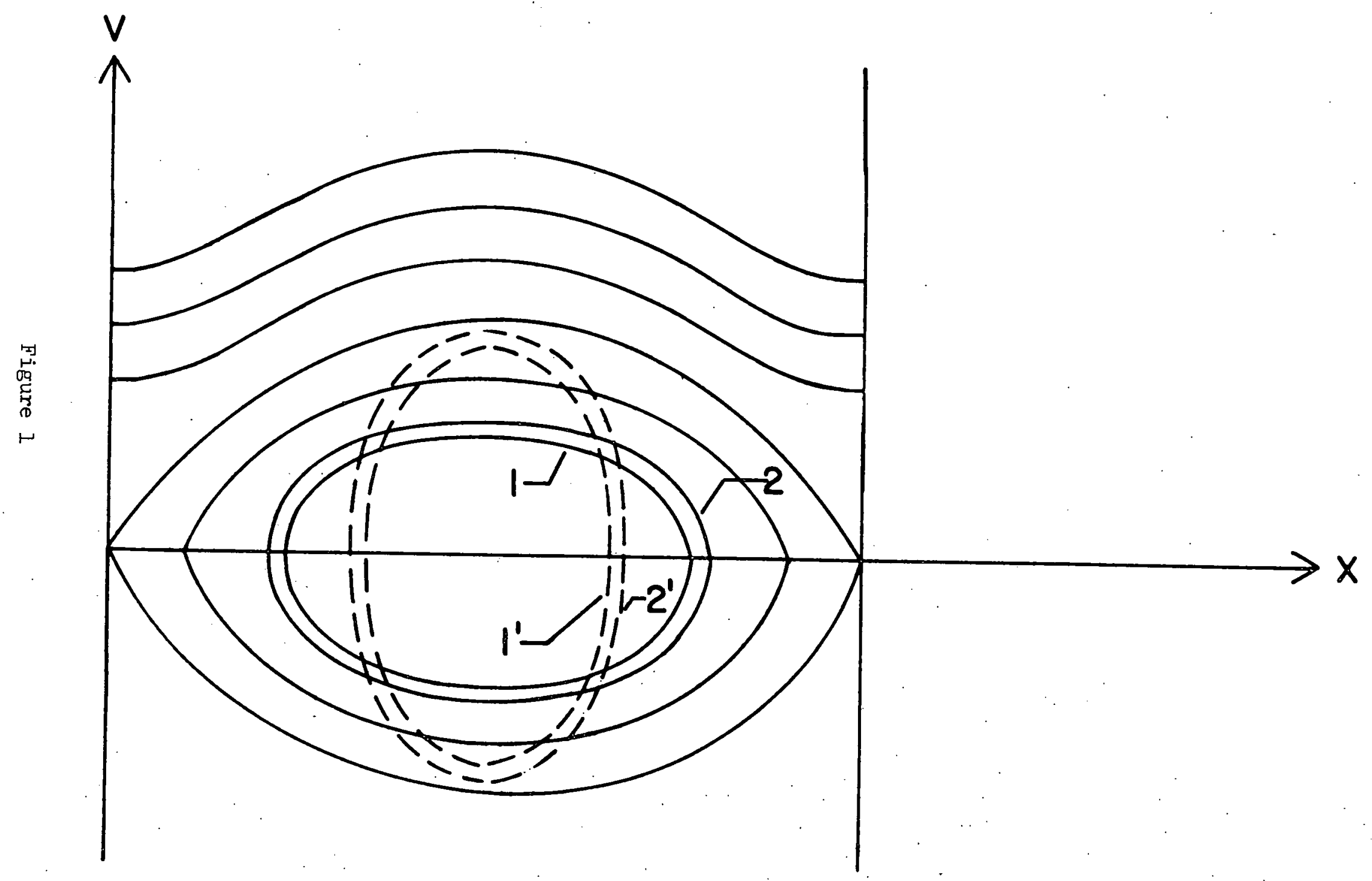

